\title{
UFIR Filtering for GPS-Based Tracking over WSNs with Delayed and Missing Data
}

\author{
Karen Uribe-Murcia, Yuriy S. Shmaliy $(\mathbb{D}$, and Jose A. Andrade-Lucio $\mathbb{C}$ \\ Department of Electronics Engineering, Universidad de Guanajuato, 36885 Salamanca, GTO, Mexico \\ Correspondence should be addressed to Yuriy S. Shmaliy; shmaliy@ugto.mx
}

Received 25 December 2017; Revised 25 March 2018; Accepted 3 April 2018; Published 8 May 2018

Academic Editor: Sos Agaian

Copyright (C) 2018 Karen Uribe-Murcia et al. This is an open access article distributed under the Creative Commons Attribution License, which permits unrestricted use, distribution, and reproduction in any medium, provided the original work is properly cited.

In smart cities, vehicles tracking is organized to increase safety by localizing cars using the Global Positioning System (GPS). The GPS-based system provides accurate tracking but is also required to be reliable and robust. As a main estimator, we propose using the unbiased finite impulse response (UFIR) filter, which meets these needs as being more robust than the Kalman filter (KF). The UFIR filter is developed for vehicle tracking in discrete-time state-space over wireless sensor networks (WSNs) with timestamped data discretely delayed on $k$-step-lags and missing data. The state-space model is represented in a way such that the UFIR filter, $\mathrm{KF}$, and $H_{\infty}$ filter can be used universally. Applications are given for measurement data, which are cooperatively transferred from a vehicle to a central station through several nodes with $k$-step-lags. Better tracking performance of the UFIR filter is shown experimentally.

\section{Introduction}

Accurate target tracking is one of the key problems in urban areas [1], which especially arises in smart cities design [2]. If a target is equipped with the Global Positioning System (GPS) tracker, then measurement data can be transferred to a central station through one or several nodes of a wireless sensor network (WSN) [3]. The problem which arises here is associated with information latency and missing data [4] due to the following main causes: high maneuverability of the target [1], failures in measurements [5], network congestion [3], non-line-of-sight (NLOS) problems [6, 7], and accidental loss of some collected data [8]. Furthermore, latency naturally occurs due to the limited bandwidth, finite propagation time [9], complexity of very large-scale integration and microelectromechanical systems [10], and time required to complete operations such as signal conditioning and storage [11]. In networks, communication delays go along with data loss called dropout or intermittence $[12,13]$. Also the delay between the measurement and its availability to the filter causes the problem of out-of-sequence measurement $[6,14]$.

Two basic models have been created for delayed data. The delays are assumed to be known when sensors are able to detect the delays or data are time-stamping $[15,16]$. In many other applications $[17,18]$, the delays are considered to be random. The problem becomes more complex in uncertain systems [19]. The best estimate is obtained here by combining delayed and nondelayed data with different probabilities.

The Kalman and $H_{\infty}$ state estimators are most widely used to deal with latency and associated issues [20]. The linear Kalman filter (KF) is optimal when it matches the system perfectly, noise is white Gaussian and uncorrelated, and the noise statistics are known along with the initial values. When such conditions are not obeyed, the KF may demonstrate poor performance $[13,21]$. The robust $H_{\infty}$ filter bounds the mean square error (MSE) for admissible parameter perturbations and delays $[19,22]$, which allows for minimizing errors with less information required than for the noise statistics $[20,23]$.

Another way to achieve better robustness is to process most recent finite data [24] using finite impulse response (FIR) filters [25]. Such filters have been developed during decades by many authors in signal processing [26-33] and control [34-36]. However, only a few authors have proposed FIR solutions for models with delays [37-40]. Let us notice that the available iterative unbiased FIR (UFIR) algorithm 
$[28,41-43]$ is most robust among other FIR solutions owing to an ability to ignore the noise statistics and initial values. This filter is bounded-input bounded-output (BIBO) stable and blind on given horizons of $N$ points, but is still not developed for observations with delayed and missing data.

In this paper, we develop the UFIR filter for GPSbased vehicle tracking over WSNs with time-stamped data discretely delayed and missing data. The rest of the paper is organized as follows. In Section 2, we consider the model and formulate the problem. In Section 3, we develop the UFIR filter for observations with delayed and missing data. Section 4 discusses the estimation errors. Section 5 gives an experimental example of applications to GPS-based tracking and concluding remarks are drawn in Section 6.

\section{Tracking Model and Problem Formulation}

A typical scenario of GPS-based vehicle tracking in WSNs is sketched in Figure 1. The vehicle current coordinates are measured by the GPS tracker. The time-stamped data are transferred to a central station (CS) via one or several nodes of the WSN. Because each node may discretely delay timestamped data at least on one-step, the vehicle location is observed in CS with a time varying $k_{n}$-step-lag depending on the vehicle location and interaction with the WSN.

2.1. Tracking Model. For $k_{n} \geqslant 0$, the vehicle dynamics and its observation can be represented in discrete-time state-space as

$$
\begin{aligned}
& x_{n}=F x_{n-1}+w_{n}, \\
& y_{n}=H x_{n-k_{n}}+v_{n},
\end{aligned}
$$

where $n$ is the discrete-time index, $x_{n} \in \mathbb{R}^{K}$ is the vehicle state vector, $y_{n} \in R^{M}$ is the observation vector, $F \in \mathbb{R}^{K \times K}$ is not singular, and $H \in \mathbb{R}^{M \times K}$. All data are time-stamped, so that $k_{n}$ is known at each $n$. Regardless of the delay, the initial state $x_{n-1}$ is supposed to be known. The uncorrelated noise vectors, $w_{n} \in \mathbb{R}^{K}$ and $v_{n} \in \mathbb{R}^{M}$, are white Gaussian with known covariances, $Q=E\left\{w_{n} w_{n}^{T}\right\}$ and $R=E\left\{v_{n} v_{n}^{T}\right\}$, and the property $E\left\{w_{n} v_{q}^{T}\right\}=0$ for all $n$ and $q$.

The UFIR filter can be applied if to transform model (1)(2) to have no latency. That can be done if to represent $x_{n-k_{n}}$ using (1) via $x_{n}$ as

$$
x_{n-k_{n}}=F^{-k_{n}}\left(x_{n}-\sum_{i=0}^{k_{n}-1} F^{i} w_{n-i}\right)
$$

and then substitute (3) into (2) and arrive at

$$
y_{n}=\bar{H}_{n} x_{n}+\bar{v}_{n}
$$

where

$$
\begin{aligned}
& \bar{H}_{n}=H F^{-k_{n}}, \\
& \bar{v}_{n}=v_{n}-H \sum_{i=0}^{k_{n}-1} F^{-k_{n}+i} w_{n-i}
\end{aligned}
$$

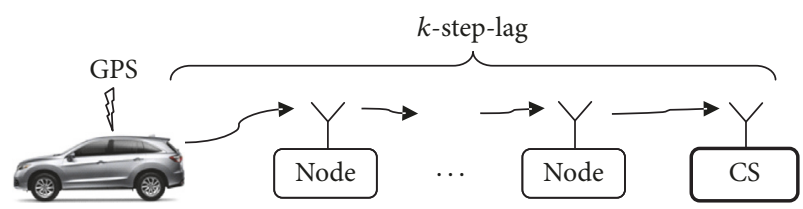

FIGURE 1: Transferring the time-stamped vehicle coordinates measured by a GPS tracker to a central station (CS) via several nodes of a WSN. Latency with a $k_{n}$-step-lag is caused by delays in the node.

and the covariance $\bar{R}_{n}=E\left\{\bar{v}_{n} \bar{v}_{n}^{T}\right\}$ of $\bar{v}_{n}$ is given by

$$
\bar{R}_{n}=R+\bar{H}_{n} \sum_{i=0}^{k_{n}-1} F^{i} Q F^{i T} \bar{H}_{n}^{T} .
$$

In compact matrix forms, (6) and (7) can be represented as

$$
\begin{aligned}
& \bar{v}_{n}=v_{n}-H \bar{B}_{n} W_{p_{n}, n}, \\
& \bar{R}_{n}=R+H \bar{B}_{n} \bar{Q}_{n} \bar{B}_{n}^{T} H^{T},
\end{aligned}
$$

where

$$
\begin{aligned}
\bar{B}_{n} & =\left[\begin{array}{llll}
F^{-1} & F^{-2} & \cdots & F^{-k_{n}}
\end{array}\right], \\
W_{p_{n}, n} & =\left[\begin{array}{llll}
w_{p_{n}}^{T} & w_{p_{n}+1}^{T} & \cdots & w_{n}^{T}
\end{array}\right]^{T},
\end{aligned}
$$

and $\bar{Q}_{n}=\operatorname{diag}\left[\begin{array}{llll}Q & Q & \cdots\end{array}\right]$ has $k_{n}$ diagonal components. Here, $\bar{B}_{\mathrm{n}}=0$ and $W_{p_{n}, n}=0$ when $k_{n}=0$ and $p_{n}>n$.

Any standard estimation technique can now be applied to models (1) and (4). However, the KF and $H_{\infty}$ filter were most developed for data with latency. Therefore, below we will introduce in brief only these filters and then compare them to the UFIR filter based on examples of tracking. We will use the following measures: $\widehat{x}_{n} \triangleq \widehat{x}_{n \mid n}$ is the estimate of $x_{n}$ over data taken from past up to and including time index $n, \widehat{x}_{n}^{-}$is the prior estimate, $P_{n}=E\left\{\left(x_{n}-\widehat{x}_{n}\right)\left(x_{n}-\widehat{x}_{n}\right)^{T}\right\}$ is the error covariance matrix, and $P_{n}^{-}=E\left\{\left(x_{n}-\widehat{x}_{n}^{-}\right)\left(x_{n}-\widehat{x}_{n}^{-}\right)^{T}\right\}$ is the prior error covariance matrix.

2.1.1. Kalman Filter. For our purposes, we will exploit an alternative form of the KF algorithm given in [44]. This algorithm starts with the prior error covariance matrix

$$
P_{n}^{-}=F P_{n-1} F^{T}+Q
$$

and then recursively updates the following values:

$$
\begin{aligned}
P_{n} & =\left(P_{n}^{-}\right)^{-1}+\bar{H}_{n}^{T} \bar{R}_{n}^{-1} \bar{H}_{n}, \\
K_{n}^{\mathrm{KF}} & =P_{n}^{-1} \bar{H}_{n}^{T} \bar{R}_{n}^{-1}, \\
\widehat{x}_{n} & =F \widehat{x}_{n-1}+K_{n}^{\mathrm{KF}}\left(y_{n}-\bar{H}_{n} F \widehat{x}_{n-1}\right), \\
P_{n+1}^{-} & =F P_{n}^{-1} F^{T}+Q,
\end{aligned}
$$

where $\bar{H}_{n}$ is given by (5) and $\bar{R}_{n}$ by (7) for any $k_{n} \geqslant 0$. 
2.1.2. $H_{\infty}$ Filter. The $H_{\infty}$ filter has been derived in [44] in the form (13) of the KF using the game theory. For $k_{n} \geqslant 0$, the $H_{\infty}$ filtering algorithm becomes

$$
\begin{aligned}
P_{n} & =\left(\check{P}_{n}^{-}\right)^{-1}-\theta_{n} S_{n}+\bar{H}_{n}^{T} \check{R}_{n}^{-1} \bar{H}_{n}, \\
K_{n}^{\infty} & =P_{n}^{-1} \bar{H}_{n}^{T} \check{R}_{n}^{-1}, \\
\widehat{x}_{n} & =F \widehat{x}_{n-1}+K_{n}^{\infty}\left(y_{n}-\bar{H}_{n} F \widehat{x}_{n-1}\right), \\
\check{P}_{n+1}^{-} & =F P_{n}^{-1} F^{T}+\check{Q},
\end{aligned}
$$

where the user-given symmetric positive definite matrices $P_{0}$, $\check{Q}$, and $\check{R}$ have different meanings than in the KF and $\check{P}_{n}^{-}$can be computed via $P_{0}$ using (12) with $Q=$ Q. To keep (14) positive definite, the positive definite matrix $S_{n} \in R^{K \times K}$ is subject to

$$
\left(\check{P}_{n}^{-}\right)^{-1}-\theta_{n} S_{n}+\bar{H}_{n}^{T} \check{R}^{-1} \bar{H}_{n}>0
$$

If equal weights are required for all errors, matrix $S_{n}$ must be set identity, $S_{n}=I$. The performance criterion for this filter is $J_{n}<1 / \theta_{n}$, in which a scalar $\theta_{n}$ must be small enough in order for the filter to be efficient. It then follows that the tuning factor $\theta_{n}$ is not allowed to be negative, even though its negative values may reduce errors when the weighting matrices are not maximized. For Gaussian noise, zero $\theta_{n}$ transforms the $H_{\infty}$ filter to the KF. For any other noise, small $\theta_{n}>0$ may result in better robustness.

The problem now formulates as follows. Given (1) and (4) with time varying $k_{n} \geqslant 0$ and missing data, we would like to develop the UFIR filter and find its fast iterative form for GPS-based tracking of a moving vehicle as shown in Figure 1. We also wish to know how the UFIR filter, KF, and $H_{\infty}$ filter measure to each other in applications to tracking.

\section{UFIR Filter for Tracking with Delayed and Missing Data}

To develop the UFIR filter for $k_{n}>0$, we extend models (1) and (4) on a horizon $[m, n]$ of $N$ points, from $m=n-N+1$ to $n$ that referring to [43] yields

$$
\begin{aligned}
& X_{m, n}=A_{N} x_{m}+B_{N} W_{m, n}, \\
& Y_{m, n}=C_{m, n} x_{m}+G_{m, n} W_{m, n}+V_{m, n}
\end{aligned}
$$

with the following extended vectors and matrices:

$$
\begin{aligned}
& X_{m, n}=\left[\begin{array}{llll}
x_{m}^{T} & x_{m+1}^{T} & \cdots & x_{n}^{T}
\end{array}\right]^{T}, \\
& Y_{m, n}=\left[\begin{array}{llll}
y_{m}^{T} & y_{m+1}^{T} & \cdots & y_{n}^{T}
\end{array}\right]^{T},
\end{aligned}
$$

$$
\begin{aligned}
& V_{m, n}=\left[\begin{array}{c}
v_{m}-C \bar{B}_{n} W_{p_{m}, m} \\
v_{m+1}-C \bar{B}_{n} W_{p_{m+1}, m+1} \\
\vdots \\
v_{n-1}-C \bar{B}_{n} W_{p_{n-1}, n-1} \\
v_{n}-C \bar{B}_{n} W_{p_{n}, n}
\end{array}\right] \text {, } \\
& A_{N}=\left[\begin{array}{llll}
I & F^{T} & \cdots & F^{N-1}{ }^{T}
\end{array}\right]^{T}, \\
& B_{N}=\left[\begin{array}{ccccc}
I & 0 & \cdots & 0 & 0 \\
F & I & \cdots & 0 & 0 \\
\vdots & \vdots & \ddots & \vdots & \vdots \\
F^{N-2} & F^{N-3} & \cdots & I & 0 \\
F^{N-1} & F^{N-2} & \cdots & F & I
\end{array}\right] \text {, } \\
& C_{m, n} \triangleq C_{m, n}(k)=\bar{C}_{m, n}(k) A_{N} \text {, } \\
& G_{m, n} \triangleq G_{m, n}(k)=\bar{C}_{m, n}(k) B_{N},
\end{aligned}
$$

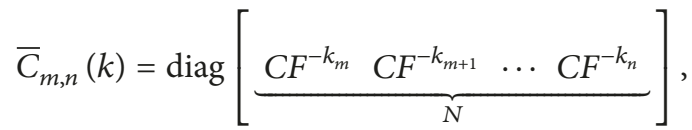

where $k$ represents a set of $\left\{k_{m}, k_{m+1}, \ldots, k_{n}\right\}$.

3.1. Batch UFIR Filter Form. The UFIR filtering estimate $\widehat{x}_{n}$ of the vehicle state $x_{n}$ can be obtained at $n$ in the batch form as $[27,34]$

$$
\widehat{x}_{n}=\mathscr{H}_{m, n} Y_{m, n},
$$

where $\mathscr{H}_{m, n}$ is the UFIR filter gain and $Y_{m, n}$ is a vector of real data (22), if to satisfy the unbiasedness condition

$$
E\left\{x_{n}\right\}=E\left\{\widehat{x}_{n}\right\},
$$

in which $E\{z\}$ means averaging of $z$ and $x_{n}$ can be represented with the last row vector in (19) as

$$
x_{n}=F^{N-1} x_{m}+B_{N}^{(N)} W_{m, n},
$$

where $B_{N}^{(N)}$ is the $N$ th row vector in (25) given by

$$
B_{N}^{(N)}=\left[\begin{array}{lllll}
F^{N-1} & F^{N-2} & \cdots & F & I
\end{array}\right] .
$$

By combining (29)-(32) and following [27], one arrives at the unbiasedness constraint

$$
I=\mathscr{H}_{m, n} \mathscr{C}_{m, n},
$$

in which

$$
\mathscr{C}_{m, n} \triangleq \mathscr{C}_{m, n}(k)=\left[\begin{array}{c}
C F^{-N+1-k_{m}} \\
\vdots \\
C F^{-1-k_{n-1}} \\
C F^{-k_{n}}
\end{array}\right]
$$


Now multiplying the both sides of (33) with $\left(\mathscr{C}_{m, n}^{T} \mathscr{C}_{m, n}\right)^{-1} \mathscr{C}_{m, n}^{T} \mathscr{C}_{m, n}$ yields the UFIR filter gain

$$
\mathscr{H}_{m, n}=\left(\mathscr{C}_{m, n}^{T} \mathscr{C}_{m, n}\right)^{-1} \mathscr{C}_{m, n}^{T}
$$

and the batch UFIR filtering estimate becomes

$$
\begin{aligned}
\widehat{x}_{n} & =\left(\mathscr{C}_{m, n}^{T} \mathscr{C}_{m, n}\right)^{-1} \mathscr{C}_{m, n}^{T} Y_{m, n} \\
& =G_{n} \mathscr{C}_{m, n}^{T} Y_{m, n}
\end{aligned}
$$

where the generalized noise power gain (GNPG) [43] is

$$
G_{n}=\mathscr{H}_{m, n} \mathscr{H}_{m, n}^{T}=\left(\mathscr{C}_{m, n}^{T} \mathscr{C}_{m, n}\right)^{-1} .
$$

The batch form (36a) may not suite real-time tracking and we go on with its fast iterative algorithm.

3.2. Iterative UFIR Filter Form. Provided (1) and (4), the standard iterative UFIR filtering algorithm [25] can be applied straightforwardly, if to substitute matrix $H$ with $\bar{H}$ given by (5). The UFIR filtering estimate (36a) can then be computed iteratively using recursions

$$
\begin{aligned}
G_{l} & =\left[\bar{H}_{l}^{T} \bar{H}_{l}+\left(F G_{l-1} F^{T}\right)^{-1}\right]^{-1}, \\
\widehat{x}_{l} & =F \widehat{x}_{l-1}+G_{l} \bar{H}_{l}^{T}\left(y_{l}-\bar{H}_{l} F \widehat{x}_{l-1}\right),
\end{aligned}
$$

beginning with $l=m+K$ and ending when $l=n$. The initial values for (38) and (39) are obtained at $s=m+K-1$ in the batch forms as

$$
\begin{aligned}
G_{s} & =\left(\mathscr{C}_{m, s}^{T} \mathscr{C}_{m, s}\right)^{-1}, \\
\tilde{x}_{s} & =G_{s} \mathscr{C}_{m, s}^{T} Y_{m, s},
\end{aligned}
$$

where $Y_{m, s}$ is a vector (22) of real data. When some data are lost, inaccurate, or unavailable, $y_{n}$ can be predicted as $y_{n}=$ $H F \widehat{x}_{n-1}$, in which case first data on the horizon $[0, N-1]$ must be available.

It is known that the linear UFIR filter is BIBO stable and not prone to divergence. However, latency in information delivery may require an ability to predict lost values that inevitably cause extra tracking errors, which we will consider next.

\section{Tracking Errors Caused by Latency, $k_{n} \geqslant 0$}

Error produced by the UFIR tracker can be defined as $\epsilon_{n}=$ $x_{n}-\widehat{x}_{n}$, where $x_{n}$ is specified by (31). Provided $\epsilon_{n}$, the error covariance matrix $P_{n}=E\left\{\epsilon_{n} \epsilon_{n}^{T}\right\}$ can also be represented in two forms.

In the batch form, matrix $P_{n}$ appears if to substitute $x_{n}$ with (31) and employ $\widehat{x}_{n}=\mathscr{H}_{m, n} Y_{m, n}$ with $Y_{m, n}$ given by (20). That yields

$$
\begin{aligned}
P_{n}= & {\left[B_{N}^{(N)}-\mathscr{H}_{m, n} G_{m, n}\right] \bar{Q}_{N}\left[B_{m, n}^{(N)}-H_{m, n} G_{m, n}\right]^{T} } \\
& +H_{m, n} \bar{R}_{N} H_{m, n}^{T},
\end{aligned}
$$

where $\bar{Q}_{N}=\operatorname{diag}\left[\begin{array}{llll}Q & Q & \cdots & Q\end{array}\right]$ and $\bar{R}_{N}=\operatorname{diag}\left[\begin{array}{lll}R & R & \cdots\end{array}\right.$ $R$ ] are square matrices with $N$ nonzero diagonal elements. It can be shown that the deterministic case of $\bar{Q}_{N}=0$ and $\bar{R}_{N}=$ 0 makes $P_{n}=0$ and the UFIR tracker has thus the deadbeat property.

4.1. Iterative Computation of $P_{n}$. Matrix (42) can also be computed iteratively, if to substitute $x_{n}$ with (1) and $\widehat{x}_{n}$ with (39). Provided the averaging, the recursion for (42) can be found as

$$
\begin{aligned}
P_{l}= & \left(I-G_{l} \bar{H}_{l}^{T} \bar{H}_{l}\right) P_{l}^{-}(\cdots)^{T}+G_{l} \bar{H}_{l}^{T} \bar{H}_{l} \\
& \times\left(\sum_{i=0}^{k_{l}-1} F^{i} Q F^{i}\right) \bar{H}_{l}^{T} \bar{H}_{l} G_{l}+G_{l} \bar{H}_{l}^{T} R \bar{H}_{l} G_{l},
\end{aligned}
$$

where $P_{l}^{-}$is given by (12), $l$ ranges as in (38) and (39), and $P_{n}$ is taken when $l=n$. Recursion (43) suggests that the tracking error grows with $k_{n}$, because the sum containing $Q$ grows with $k_{n}$. However, the same cannot be said about $R$, which does not accumulate the effect of $k_{n}$.

With no latency, the sum in (43) becomes identically zero and one arrives at the error covariance $P_{l}$ of the standard UFIR filter [43],

$$
P_{l}=\left(I-G_{l} H^{T} H\right) P_{l}^{-}(\cdots)^{T}+G_{l} H^{T} R H G_{l},
$$

which also holds for the KF, if to substitute $G_{n} H^{T}$ with the Kalman gain $K_{n}$.

Note that the minimization of tracking errors will require an optimal number $N_{\text {opt }}-1$ of iterations for the UFIR filter. At the test stage, the optimal horizon $N_{\text {opt }}$ can be found for the known ground truth $x_{n}$ by minimizing the MSE via the trace of $\mathbf{P}_{n}$ [30] depicted as $\operatorname{tr} \mathbf{P}_{n}$. Because the ground truth is unavailable in real tracking, $N_{\text {opt }}$ can be estimated via the measurement residual as shown in [30].

\section{GPS-Based Tracking of a Moving Vehicle}

We will now consider the case shown in Figure 1 when the GPS tracker measures the vehicle coordinates of location at each time index $n$ and transfer time-stamped data cooperatively to a CS of a WSN via one or several nodes. We admit that each node may introduce latency and data will thus arrive at the CS with known delay on $k_{n}>0$ points. At different time instances, a vehicle may interact with a different number of the nodes that will make the $k_{n}$-step-lag time varying. We will base our investigations on data obtained in the Cook county of Illinois and available for free use from the University of Illinois at Chicago. To simplify the problem, in this paper we will consider the case of a constant latency, $k=k_{n}$.

Concerned with the tracking errors and not with the actual vehicle location, we will conventionally place the start point at zero coordinates as shown in Figure 2.

5.1. State-Space Model. To investigate the trade-off between the estimators, we will suppose that a vehicle is represented with two states in each directions and assign the state vector, 


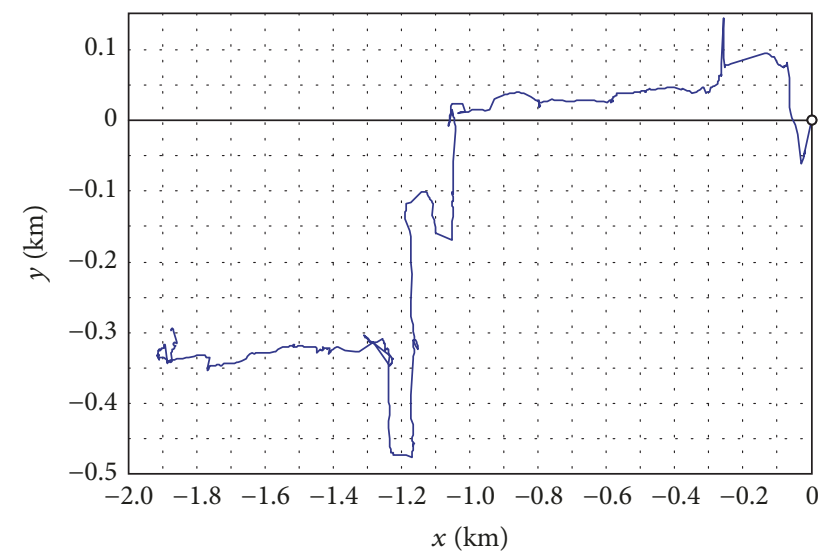

FIGURE 2: GPS-based vehicle trajectory measured in the north $(y)$ and east $(x)$ coordinates, both in $\mathrm{km}$, with the start point at $\{0,0\}$. Measurements are provided each second at 858 data points.

$K=4$, as $x_{n}=\left[\begin{array}{llll}x_{1 n} & x_{2 n} & x_{3 n} & x_{4 n}\end{array}\right]^{T}$, where $x_{1 n}=x_{n}, x_{2 n}=$ $\dot{x}_{n}, x_{3 n}=y_{n}$, and $x_{4 n}=\dot{y}_{n}$. Accordingly, the system matrix attains the form of

$$
F=\left[\begin{array}{llll}
1 & \tau & 0 & 0 \\
0 & 1 & 0 & 0 \\
0 & 0 & 1 & \tau \\
0 & 0 & 0 & 1
\end{array}\right]
$$

where the sampling time is $\tau=1 \mathrm{~s}$ for the considered database. The GPS tracker provides measurements of the vehicle coordinates, $x$ and $y$. Therefore, the measurement matrix is

$$
H=\left[\begin{array}{llll}
1 & 0 & 0 & 0 \\
0 & 0 & 1 & 0
\end{array}\right]
$$

Provided $F$ and $H$, matrix $\mathscr{C}_{m, s}$ required by the UFIR filter to compute the initial values (40) and (41) for $s=m+3$ becomes

$$
\mathscr{C}_{s, m}=\left[\begin{array}{cccc}
1 & -3 \tau & 0 & 0 \\
0 & 0 & 1 & -3 \tau \\
1 & -2 \tau & 0 & 0 \\
0 & 0 & 1 & -2 \tau \\
1 & -\tau & 0 & 0 \\
0 & 0 & 1 & -\tau \\
1 & 0 & 0 & 0 \\
0 & 0 & 1 & 0
\end{array}\right] F^{-k} \text {. }
$$

The only tuning factor $N_{\text {opt }}=5$ required by the UFIR filter was found for $k=0$ by minimizing the derivative of the trace of the mean square value of the residual $y_{n}-H \widehat{x}_{n}(N)$, as shown in [30]. Because $N_{\text {opt }}$ depends on $k$, we will apply $N_{\text {opt }}=5$ in the worst case for the UFIR filter.

Having no information about the process noise, we observe similar trajectories and estimate the average vehicle

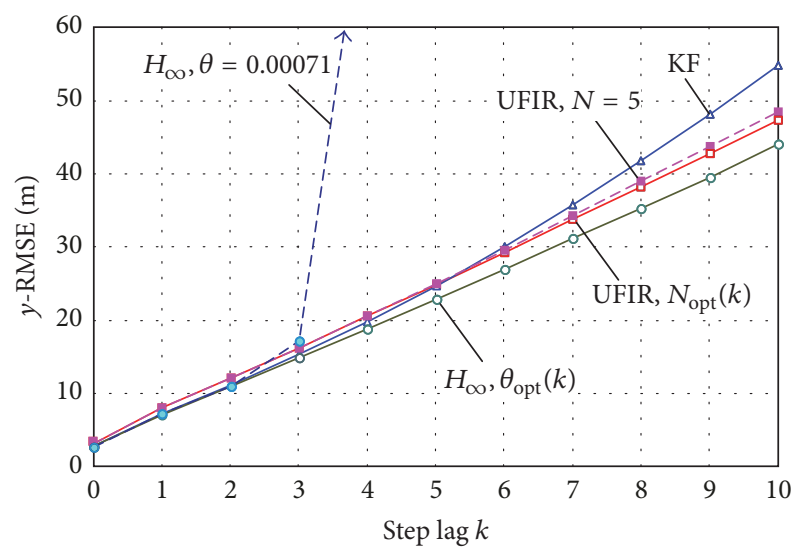

FIGURE 3: Effect of the $k$-step-lag on the $y$-RMSE of the UFIR filter, $\mathrm{KF}$, and $H_{\infty}$ filter with different tunings.

speed by about $10 \mathrm{~m} / \mathrm{s}$ or $36 \mathrm{~km} /$ hour. Next, accepting the speed standard deviation of about $20 \%$, we set $\sigma_{w 2}=2 \mathrm{~m} / \mathrm{s}$ to the second state along each of the coordinates, ignore the unknown noise in the first state, $\sigma_{w 1}=0$, and describe matrix $Q$ as

$$
Q=\sigma_{w 2}^{2}\left[\begin{array}{cccc}
\tau^{2} / 2 & \tau / 2 & 0 & 0 \\
\tau / 2 & 1 & 0 & 0 \\
0 & 0 & \tau^{2} / 2 & \tau / 2 \\
0 & 0 & \tau / 2 & 1
\end{array}\right]
$$

The GPS standard positioning service provides navigation with an error of less than 15 meters with the probability of $95 \%$ in the 2-sigma sense. Referring to this value, we assign the standard deviation of the measurement noise in each direction as $\sigma_{v}=15 / 4=3.75 \mathrm{~m}$ and obtain

$$
R=\left[\begin{array}{cc}
\sigma_{v}^{2} & 0 \\
0 & \sigma_{v}^{2}
\end{array}\right] .
$$

Because the above provided matrices $Q$ and $R$ are overestimated, we set $\check{Q}=Q$ and $\check{R}=R$ for $H_{\infty}$. It is expected that the tuning factor $\theta$ will improve the performance of the $H_{\infty}$ filter by minimizing the MSE for the maximized errors. However, the ground truth is not available in tracking. Therefore, we will find $\theta$ for the measured trajectory and consider it as the best case for $H_{\infty}$, which is unfeasible.

5.2. Effect of Latency on the Estimation Accuracy. We start with learning the effect of $k$ on the estimation accuracy, which is illustrated in Figure 3 with the root MSEs (RMSEs) in the north direction ( $y$-RMSE). The KF is self-tuned to $k$. Therefore, we consider its RMSE as a benchmark. A special feature of the KF is that the RMSE grows with $k$ nonlinearly and faster than in the UFIR and $H_{\infty}$ filters.

The UFIR filter produces a bit more errors than in the $\mathrm{KF}$ with small $k$ and lesser with larger $k$. A special feature is that the UFIR estimate is of low sensitivity to $N$, in which 


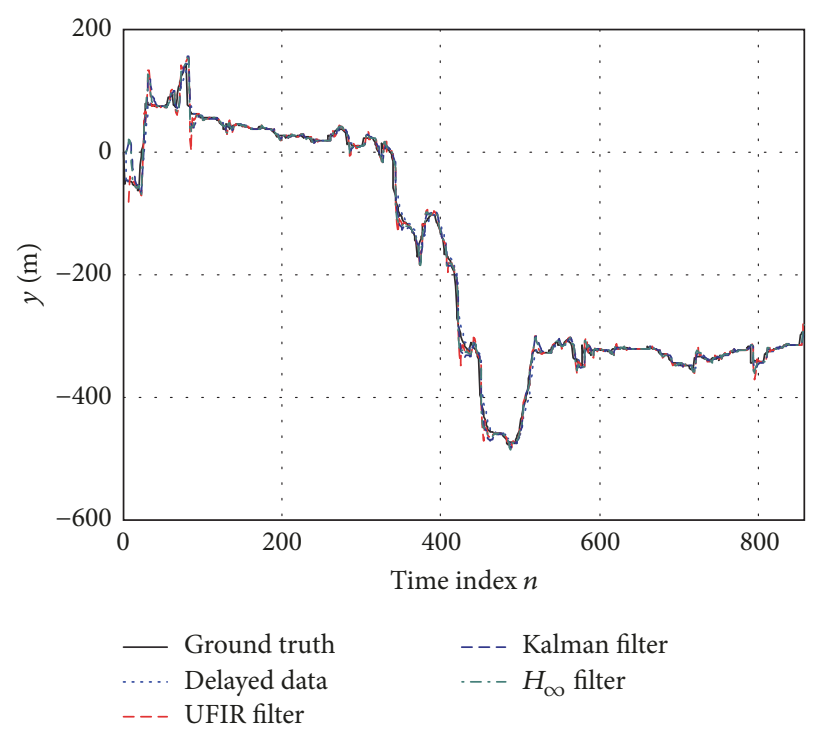

(a)
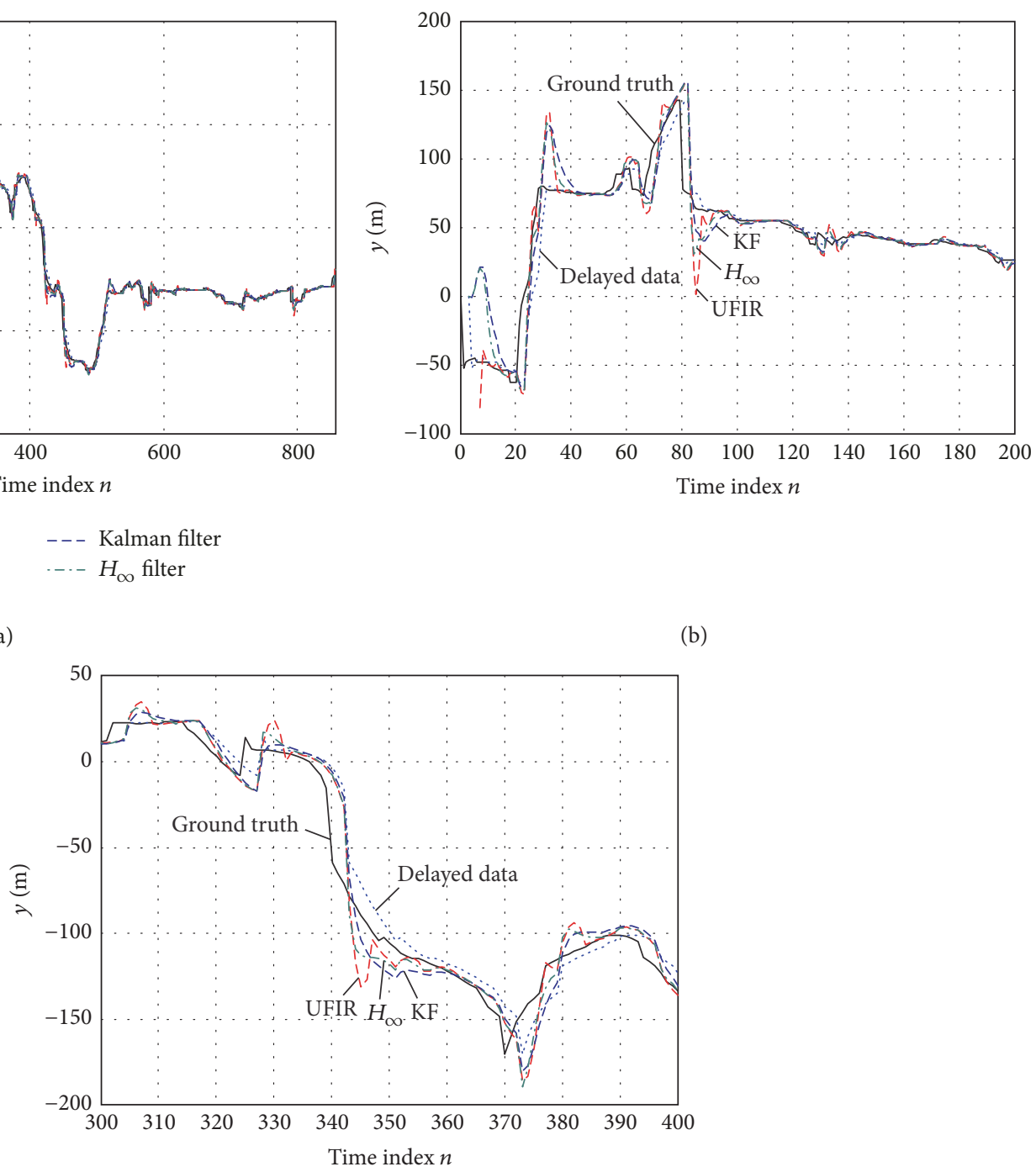

(c)

FIGURE 4: Tracking in the north direction $y, m$ for $k=3$ with UFIR filter $\left(N_{\text {opt }}=5\right)$, KF, and $H_{\infty}$ filter $\left(\theta=3.3 \times 10^{-4}\right)$ : (a) full time scale, (b) $0 \leqslant n \leqslant 200$, and (c) $300 \leqslant n \leqslant 400$.

optimal value $N_{\text {opt }}=5$ holds for $0 \leqslant k \leqslant 4$, increases to $N_{\text {opt }}=6$ for $5 \leqslant k \leqslant 6$, and reaches $N_{\text {opt }}=7$ when $7 \leqslant k \leqslant 10$. Of practical importance is that setting $N$ optimally for each $k$ does not improve the performance essentially against the worst case when $N_{\text {opt }}=5$ is set for all $k$.

The $H_{\infty}$ filter outperforms both the UFIR filter and KF, provided that $\theta$ is set properly for each lag $k$. However, this filter is highly sensitive to $\theta$, in which optimal value $\theta_{\text {opt }}$ ranges from $1.8 \times 10^{-2}$ for $k=0$ to $1.066 \times 10^{-5}$ for $k=10$ in a nonlinear way. Unlike in the UFIR filter, a constant $\theta$ is unacceptable for all $k$. An example is given in Figure 3, where $\theta_{\text {opt }}=7.1 \times 10^{-4}$ found for $k=2$ is applied in a wide range of $k$. As can be seen, it is only when $k=2$ that the $H_{\infty}$ filter improves the KF performance. For $k<2$, there is no improvement and, when $k>2$, the $H_{\infty}$ filter rapidly diverges.
5.3. Tracking over Data Delayed on $k=3$. We now suppose that data are transferred from a vehicle to a CS with $k=3$ and investigate tracking errors in the north and east directions. Filters will be tuned as follows: UFIR in the worst case of $N_{\text {opt }}=5$ being valid for $k=0 ; \mathrm{KF}$ as near optimal; and $H_{\infty}$ being in the best (unfeasible) case of known ground truth.

5.3.1. Tracking in the North Direction. The vehicle trajectory (measured and delayed) and estimates provided by the filters in the north direction are sketched in Figure 4. As can be seen in Figure 4(a), the trajectory is nonlinear and the vehicle sometimes maneuvers rapidly. All filters produce consistent estimates with poorly distinguishable differences (Figure 4(a)). We therefore additionally show two parts of the trajectory on short-time spans of $0 \leqslant n \leqslant 200$ in Figure 4(b) and $300 \leqslant n \leqslant 400$ in 


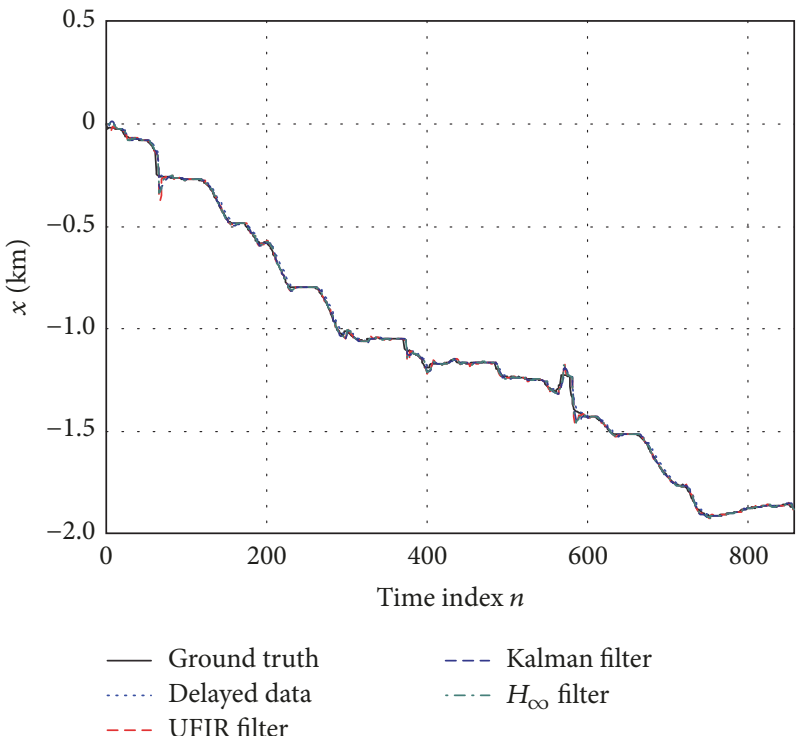

(a)

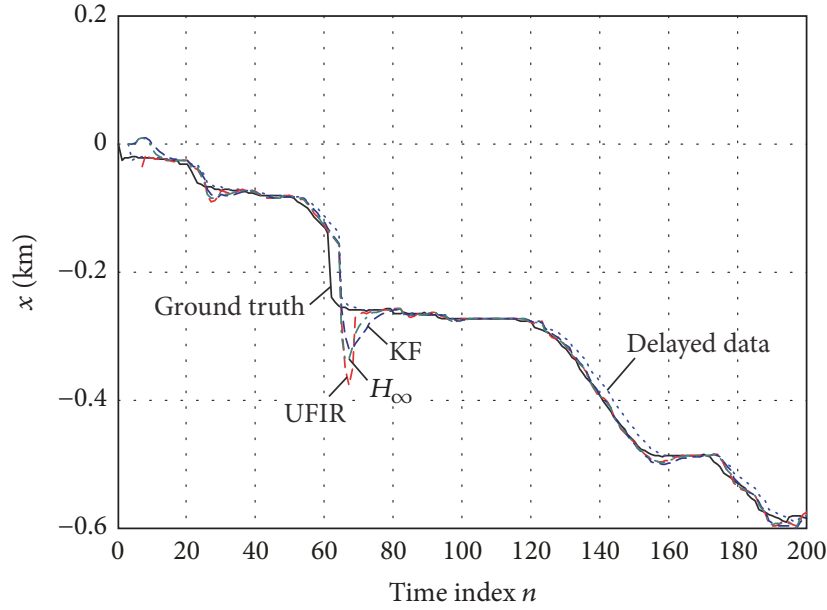

(b)

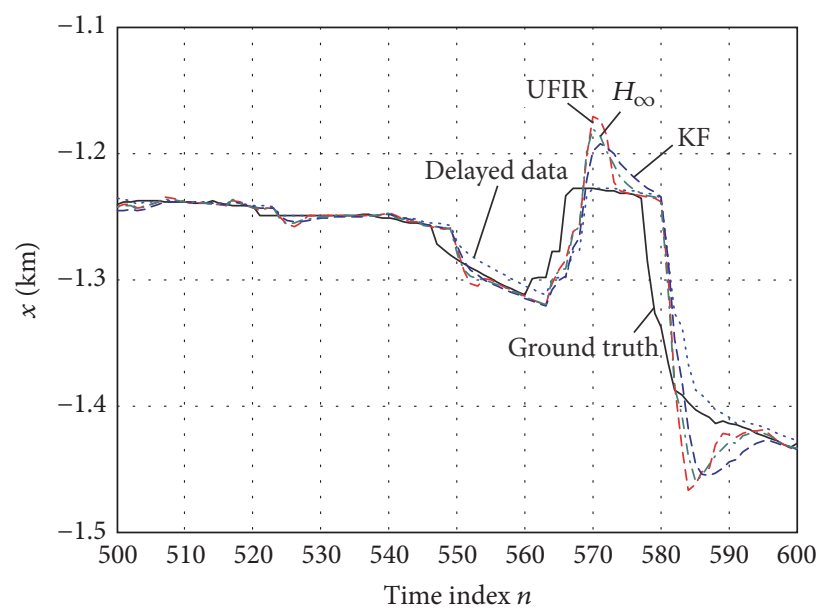

(c)

FIGURE 5: Tracking in the east direction $x, m$ for $k=3$ with UFIR filter $\left(N_{\mathrm{opt}}=5\right)$, KF, and $H_{\infty}$ filter $\left(\theta=3.3 \times 10^{-4}\right)$ : (a) full time scale, (b) $0 \leqslant n \leqslant 200$, and (c) $500 \leqslant n \leqslant 600$.

Figure 4(c). Several observations can be made from Figure 4:

(i) All filters track well the trajectory when a vehicle travels with a near constant velocity in one direction, as on $140 \leqslant n \leqslant 180$ in Figure 4(b).

(ii) Filters temporarily lose an ability of tracking and go along the delayed data when a vehicle quickly changes the direction, as on $340 \leqslant n \leqslant 343$ in Figure 4(c).

(iii) Responding to fast maneuvers, all filters produce dynamic errors such that the UFIR filter comes up with larger excursions but shorter transients, KF with shorter excursions but longer transients, and $H_{\infty}$ filter demonstrates inbetween properties; see on $80 \leqslant$ $n \leqslant 100$ in Figure 4(b) and $344 \leqslant n \leqslant 360$ in Figure 4(c).
Because errors are unacceptably large in all filters when they temporarily lose an ability of tracking, a key question arises of how fast each of them returns back to the normal mode. In this regard the UFIR filter looks better with its shortest transient.

5.3.2. Tracking in the East Direction. Tracking in the east direction (Figure 5) does not reveal any essential features. The filters still track well the trajectory when a vehicle travels with a near constant velocity as, for example, in a span of $120 \leqslant n \leqslant 160$ in Figure 5(b). Here, all filters also temporarily lose an ability of tracking and go along the delayed data when the trajectory quickly changes as, for example, in a span of $560 \leqslant n \leqslant 570$ in Figure 5(c). Finally, the UFIR filter still demonstrates larger excursions but shorter transients, KF shorter excursions but longer transients, and $H_{\infty}$ filter is inbetween as, for example, in 


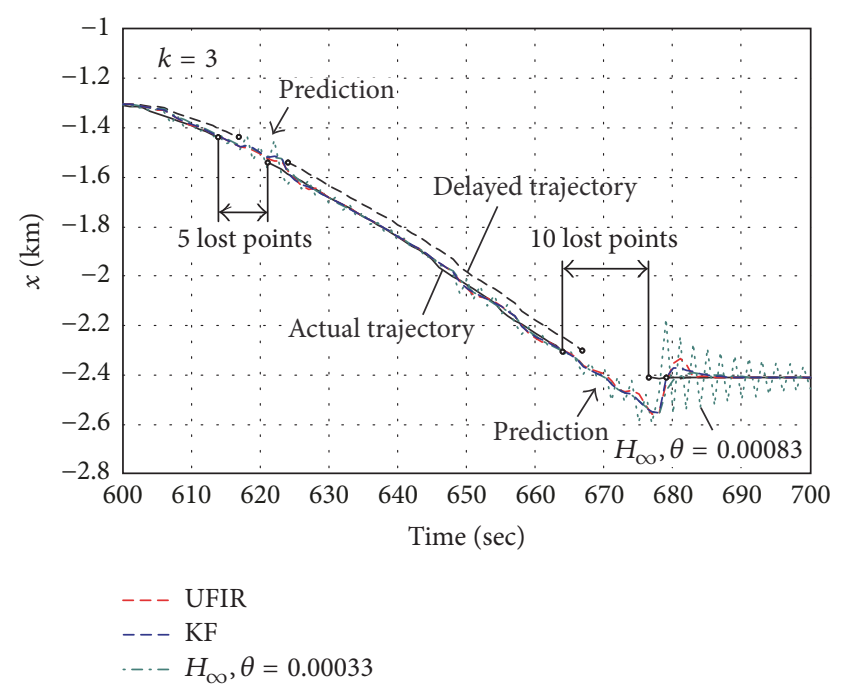

FIGURE 6: Vehicle tracking with temporary missing data in the east direction $x$ by the UFIR filter, KF, and $H_{\infty}$ filter, all augmented with the prediction option. Tuned improperly, the $H_{\infty}$ filter becomes unstable and prone to divergence, as in Figure 3.

spans of $60 \leqslant n \leqslant 80$ in Figure $5(\mathrm{~b})$ and $570 \leqslant n \leqslant 580$ in Figure 5(c).

5.4. Tracking with Temporary Lost Data. We finally admit that some data points can be lost during the transmission and remove 5 data points at $n=(615 \cdots 620) \mathrm{s}$ and 10 at $n=(665 \cdots 675) \mathrm{s}$ as shown in Figure 6 for $k=3$. To predict lost data, we augment each algorithm with the prediction block as mentioned below (41) and run the filters. As can be seen, the filters act consistently with, however, some specifics. The estimates do not get away essentially from each other and the actual trajectory. However, when a vehicle maneuvers during the prediction, all filters diverge and return back to the actual trajectory with similar transients as in Figures 4 and 5. The latter again speaks in favor of the UFIR filter, which has shorter transients.

\section{Conclusions}

The UFIR filter developed in this paper for GPS-based vehicle tracking over WSNs with time-stamped discretely delayed and missing data has demonstrated better performance than the KF and $H_{\infty}$ filter. The main benefits of using the UFIR filter are that it (1) does not require any information about noise and initial conditions, (2) becomes blind on given horizons, and (3) has shorter transients. The latter can be considered as an important practical advantage in all situations when the trajectory changes rapidly and estimators temporarily loses an ability of tracking. Applications to GPS-based vehicle tracking with known discretely delayed and missed data have proved a better performance of the UFIR filter.

\section{Conflicts of Interest}

The authors declare that there are no conflicts of interest regarding the publication of this paper.

\section{References}

[1] Y. Bar-Shalom, X. R. Li, and T. Kirubarajan, Estimation with Applications to Tracking and Navigation, John Wiley \& Sons, Hoboken, NJ, USA, 2001.

[2] H. Song, R. Srinivasan, T. Sookoor, and S. Jeschke, Smart Cities: Foundations, Principles, and Applications, Wiley, Hoboken, NJ, USA, 2017.

[3] F. Zhao and L. J. Guibas, Wireless Sensor Networks: An Information Processing Approach, Elsevier, Amsterdam, The Netherlands, 2004.

[4] M. Malek-Zavarei and M. Jamshidi, Time-Delay Systems: Analysis, Optimization and Application, North-Holland Publishing, Amsterdam, The Netherlands, 1987.

[5] H. Wang, T. Kirubarajan, and Y. Bar-Shalom, "Precision large scale air traffic surveillance using IMM/assignment estimators," IEEE Transactions on Aerospace and Electronic Systems, vol. 35, no. 1, pp. 255-266, 1999.

[6] Y. Bar-Shalom, "Update with out-of-sequence measurements in tracking: exact solution," IEEE Transactions on Aerospace and Electronic Systems, vol. 38, no. 3, pp. 769-778, 2002.

[7] S. Zhang and Y. Bar-Shalom, "Optimal update with multiple out-of-sequence measurements with arbitrary arriving order," IEEE Transactions on Aerospace and Electronic Systems, vol. 48, no. 4, pp. 3116-3132, 2012.

[8] Z. Wang, F. Yang, D. W. C. Ho, and X. Liu, "Robust $H_{\infty}$ filtering for stochastic time-delay systems with missing measurements," IEEE Transactions on Signal Processing, vol. 54, no. 7, pp. 25792587, 2006.

[9] A. Ray, "Introduction to networking for integrated control systems," IEEE Control Systems Magazine, vol. 9, no. 1, pp. 76-79, 1989.

[10] M. Sharma, R. Gautam, and M. A. Khan, Design and Modeling of Low Power VLSI Systems, IGI Global, Herchey, Pa, USA, 2016.

[11] D. C. Swanson, Signal Processing for Intelligent Sensor Systems with MATLAB, Taylor \& Francis Group, Boca Raton, Fla, USA, 2nd edition, 2011.

[12] M. Sahebsara, T. Chen, and S. L. Shah, "Optimal $H_{\infty}$ filtering in networked control systems with multiple packet dropout," IEEE Transactions on Automatic Control, vol. 52, no. 8, pp. 1508-1513, 2007.

[13] B. Sinopoli, L. Schenato, M. Franceschetti, K. Poolla, M. I. Jordan, and S. S. Sastry, "Kalman filtering with intermittent observations," Institute of Electrical and Electronics Engineers Transactions on Automatic Control, vol. 49, no. 9, pp. 1453-1464, 2004.

[14] K. Zhang, X. R. Li, and Y. Zhu, "Optimal update with out-ofsequence measurements," IEEE Transactions on Signal Processing, vol. 53, no. 6, pp. 1992-2004, 2005.

[15] T. Kailath, A. H. Sayed, and B. Hassibi, Linear Estimation, Prentice-Hall, Englewood Cliffs, NJ, USA, 1999.

[16] H. Zhang, G. Feng, G. Duan, and X. Lu, " $H_{\infty}$ filtering for multiple-time-delay measurements," IEEE Transactions on Signal Processing, vol. 54, no. 5, pp. 1681-1688, 2006.

[17] J. D. Gibson, Mobile Communications Handbook, Taylor \& Francis Group, Boca Raton, Fla, USA, 3rd edition, 2013.

[18] M. Chitre, S. Shahabudeen, and M. Stojanovic, "Underwater acoustic communications and networking: recent advances and future challenges," Marine Technology Society Journal, vol. 42, no. 1, pp. 103-116, 2008. 
[19] Z. Wang, D. W. C. Ho, and X. Liu, "Robust filtering under randomly varying sensor delay with variance constraints," IEEE Transactions on Circuits and Systems II: Express Briefs, vol. 51, no. 6, pp. 320-326, 2004.

[20] M. Liu and H. Chen, " $H{ }_{\infty}$ state estimation for discretetime delayed systems of the neural network type with multiple missing measurements," IEEE Transactions on Neural Networks and Learning Systems, vol. 26, no. 12, pp. 2987-2998, 2015.

[21] A. K. Singh, P. Date, and S. Bhaumik, "A modified Bayesian filter for randomly delayed measurements," IEEE Transactions on Automatic Control, vol. 62, no. 1, pp. 419-424, 2017.

[22] R. M. Palhares, C. E. de Souza, and P. L. Dias Peres, "Robust $H_{\infty}$ filtering for uncertain discrete-time state-delayed systems," IEEE Transactions on Signal Processing, vol. 49, no. 8, pp. 16961703, 2001.

[23] K. M. Nagpal and P. P. Khargonekar, "Filtering and smoothing in an $H_{\infty}$ setting," IEEE Transactions on Automatic Control, vol. 36, no. 2, pp. 152-166, 1991.

[24] A. H. Jazwinski, Stochastic Processes and Filtering Theory, Academic Press, New York, NY, USA, 1970.

[25] Y. S. Shmaliy, S. Zhao, and C. K. Ahn, "Unbiased finite impulse response filtering: an iterative alternative to Kalman filtering ignoring noise and initial conditions," IEEE Control Systems Magazine, vol. 37, no. 5, pp. 70-89, 2017.

[26] Y. S. Shmaliy, "Unbiased FIR filtering of discrete-time polynomial state-space models," IEEE Transactions on Signal Processing, vol. 57, no. 4, pp. 1241-1249, 2009.

[27] Y. S. Shmaliy, "Linear optimal FIR estimation of discrete timeinvariant state-space models," IEEE Transactions on Signal Processing, vol. 58, no. 6, pp. 3086-3096, 2010.

[28] Y. S. Shmaliy, "An iterative Kalman-like algorithm ignoring noise and initial conditions," IEEE Transactions on Signal Processing, vol. 59, no. 6, pp. 2465-2473, 2011.

[29] Y. S. Shmaliy, "Suboptimal FIR filtering of nonlinear models in additive white Gaussian noise," IEEE Transactions on Signal Processing, vol. 60, no. 10, pp. 5519-5527, 2012.

[30] F. Ramirez-Echeverria, A. Sarr, and Y. Shmaliy, "Optimal memory for discrete-time FIR filters in state-space," IEEE Transactions on Signal Processing, vol. 62, no. 3, pp. 557-561, 2014.

[31] S. Zhao, Y. S. Shmaliy, F. Liu, and S. H. Khan, "Unbiased, optimal, and in-betweens: The trade-off in discrete finite impulse response filtering," IET Signal Processing, vol. 10, no. 4, pp. 325334, 2016.

[32] S. Zhao, Y. S. Shmaliy, and F. Liu, "Fast Kalman-like optimal unbiased FIR filtering with applications," IEEE Transactions on Signal Processing, vol. 64, no. 9, pp. 2284-2297, 2016.

[33] S. Zhao, Y. S. Shmaliy, and F. Liu, "Fast computation of discrete optimal FIR estimates in white Gaussian noise," IEEE Signal Processing Letters, vol. 22, no. 6, pp. 718-722, 2015.

[34] W. H. Kwon and S. Han, Receding Horizon Control: Model Predictive Control for State Models, Springer, London, UK, 2005.

[35] Z. Quan, S. Han, and W. H. Kwon, "A robust FIR filter for linear discrete-time state-space signal models with uncertainties," IEEE Signal Processing Letters, vol. 14, no. 8, pp. 553-556, 2007.

[36] C. K. Ahn, "Strictly passive FIR filtering for state-space models with external disturbance," International Journal of Electronics and Communications, vol. 66, no. 11, pp. 944-948, 2012.

[37] C. K. Ahn, P. Shi, and L. Wu, "Receding horizon stabilization and disturbance attenuation for neural networks with timevarying delay," IEEE Transactions on Cybernetics, vol. 45, no. 12, pp. 2680-2692, 2015.
[38] I. Y. Song, D. Y. Kim, V. Shin, and M. Jeon, "Receding horizon filtering for discrete-time linear systems with state and observation delays," IET Radar, Sonar \& Navigation, vol. 6, no. 4, pp. 263-271, 2012.

[39] I. Y. Song, V. Shin, and M. Jeon, "Distributed fusion receding horizon filtering for uncertain linear stochastic systems with time-delay sensors," Journal of The Franklin Institute, vol. 349, no. 3, pp. 928-946, 2012.

[40] H. D. Choi, C. K. Ahn, M. T. Lim, and M. K. Song, "Dynamic output-feedback $H_{\infty}$ control for active half-vehicle suspension systems with time-varying input delay," International Journal of Control, Automation, and Systems, vol. 14, no. 1, pp. 59-68, 2016.

[41] Y. S. Shmaliy, S. H. Khan, S. Zhao, and O. Ibarra-Manzano, "General unbiased FIR filter with applications to GPS-based steering of oscillator frequency," IEEE Transactions on Control Systems Technology, vol. 25, no. 3, pp. 1141-1148, 2017.

[42] Y. S. Shmaliy, S. Khan, and S. Zhao, "Ultimate iterative UFIR filtering algorithm," Measurement, vol. 92, pp. 236-242, 2016.

[43] Y. S. Shmaliy and D. Simon, "Iterative unbiased FIR state estimation: a review of algorithms," EURASIP Journal on Advances in Signal Processing, vol. 2013, article 113, 16 pages, 2013.

[44] D. Simon, Optimal State Estimation: Kalman, $H_{\infty}$, and Nonlinear Approaches, J. Wiley \& Sons, Hoboken, NJ, USA, 2006. 


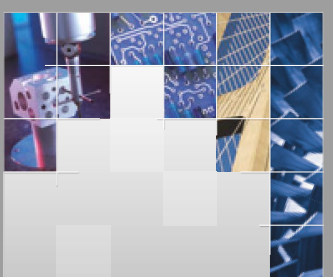

\section{Enfincering}
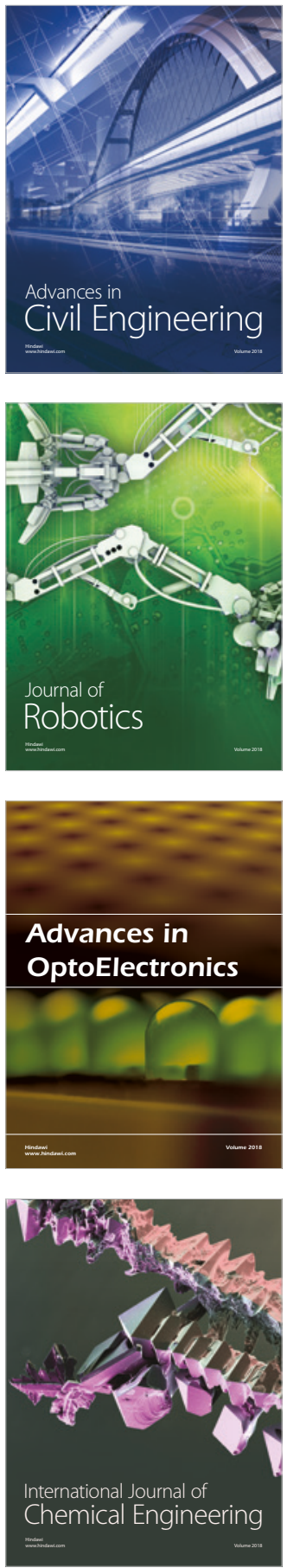

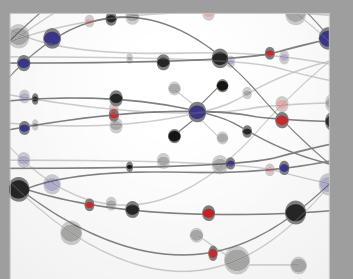

\section{Rotating \\ Machinery}

The Scientific World Journal

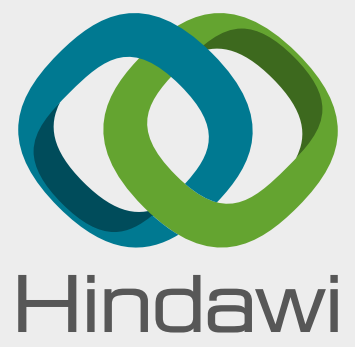

Submit your manuscripts at

www.hindawi.com
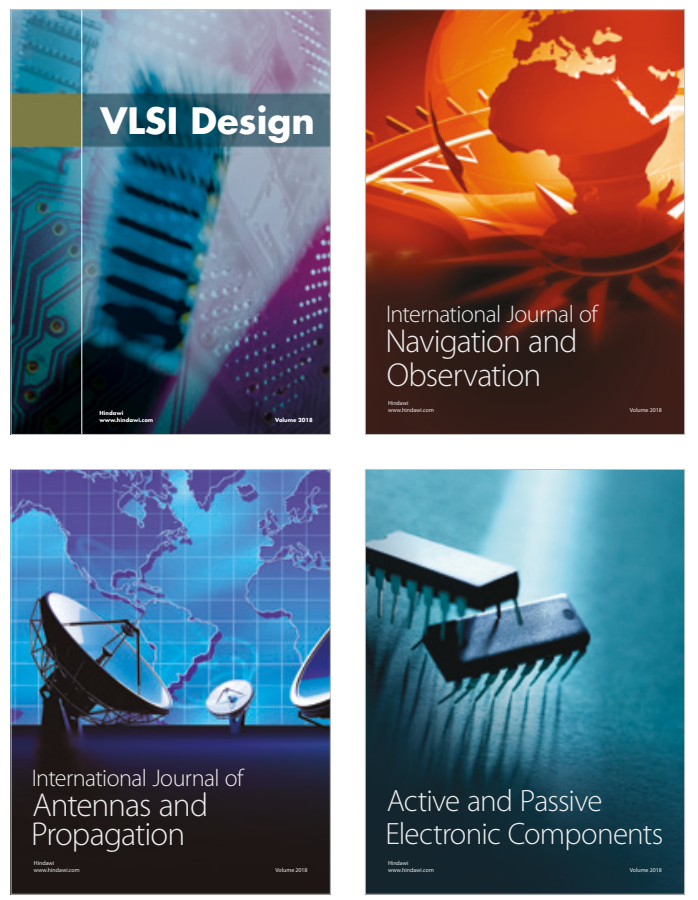
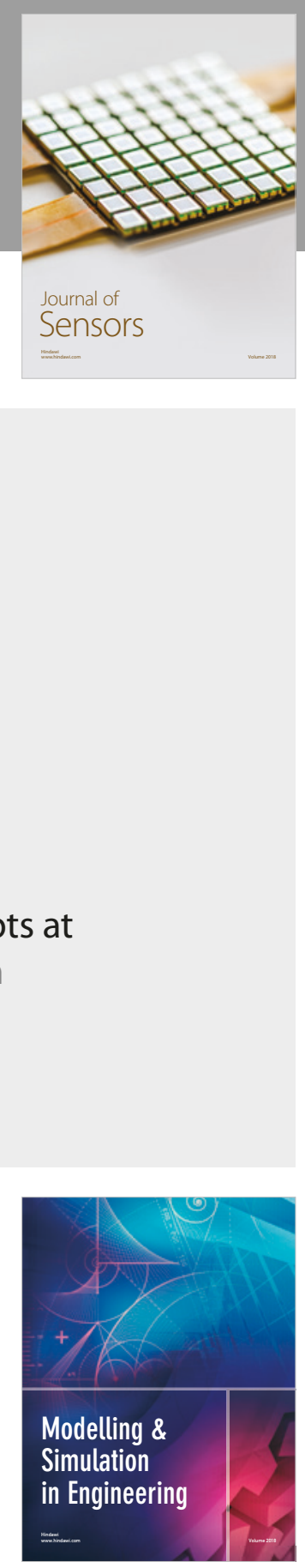

\section{Advances \\ Multimedia}
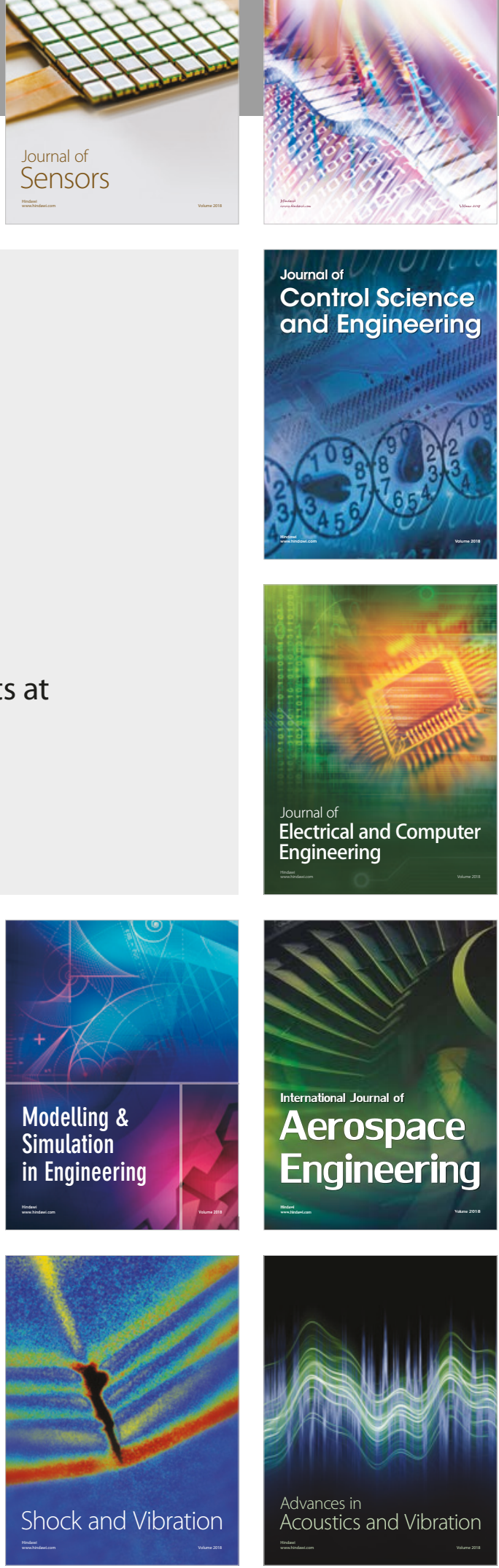